\title{
Trastuzumab-associated autoimmune thyroid disease in a patient with metastatic breast cancer
}

Hong Ki Min, In-Ho Kim, Jae Young Kim, Tae-hyun Ban, Kyung Hoon Kim, Jeonghoon Ha, and Jung-Hoon Kim

Division of Oncology, Department of Internal Medicine, College of Medicine, The Catholic University of Korea, Seoul, Korea

Received: February 24, 2014

Revised : August 16, 2014 Accepted: December 26, 2014

\section{Correspondence to}

Jung-Hoon Kim, M.D.

Division of Oncology, Department of Internal Medicine, College of Medicine, Incheon St. Mary's Hospital, The Catholic University of Korea, 56 Dongsu-ro,

Bupyeong-gu, Incheon 21431, Korea

Tel: +82-32-280-5969

Fax: +82-32-280-5987

E-mail: willgraham@naver.com
To the Editor,

The cell surface extracellular domain of human epidermal growth factor receptor 2 (HER-2) serves as a checkpoint for cell proliferation and differentiation. HER-2 expression is associated with tumor aggressiveness in breast cancer. Trastuzumab is a humanized monoclonal antibody (MAB) directed against HER-2, and trastuzumab alone or in combination with conventional chemotherapy is the standard treatment for breast cancer patients whose tumors overexpress HER-2. Although trastuzumab is generally well tolerated, a small number of patients develop mild adverse reactions to this treatment. Among such reactions, autoimmune thyroiditis is a rare adverse event associated with trastuzumab infusion, with an incidence rate of $0.3 \%$. To date, four cases of trastuzumab-associated autoimmune thyroid disease (AITD) have been recorded in adjuvant breast cancer studies; however, details of these cases, including clinical manifestations of disease severity and their natural courses, were not reported. Here we report a case of trastuzumab-associated AITD that developed 3 days after the first cycle of trastuzumab therapy in a metastatic breast cancer patient.

A 42-year-old female patient was hospitalized due to headaches and back pain. The patient had undergone mod- ified radical mastectomy on her left breast 2 years prior, followed by eight rounds of adriamycin and cyclophosphamide-based chemotherapy. Examination of the surgical breast tissue revealed an overexpression of HER-2. Five months prior to her hospitalization, an annual imaging study revealed the presence of metastases at multiple sites. However, the metastatic breast cancer was not treated because the patient refused treatment.

A general examination indicated that she was afebrile and tachycardic. On physical examination, her skin color and bilateral conjunctivae showed jaundice, and mild hepatomegaly with abdominal bloating was noted. Several bilateral lymph nodes with firm texture were palpable in the cervical area. The thyroid gland was normal in size and texture. A respiratory system examination revealed diminished breath sounds in bilateral lower lung fields. A chest X-ray showed bilateral costophrenic blunting. Laboratory tests, including complete blood counts and blood chemistries, were performed. The results were as follows: hemoglobin, $12.2 \mathrm{mg} / \mathrm{dL}$; leukocyte count, 11.87 $\times 10^{9}$ cells/L $(82.6 \%$ neutrophils, $9.6 \%$ lymphocytes, $6.8 \%$ monocytes, $0.7 \%$ eosinophils, and $0.3 \%$ basophils); platelet count, $220 \times 10^{9}$ cells $/ \mathrm{L}$; serum aspartate aminotransferase, $254 \mathrm{IU} / \mathrm{L}$; serum 


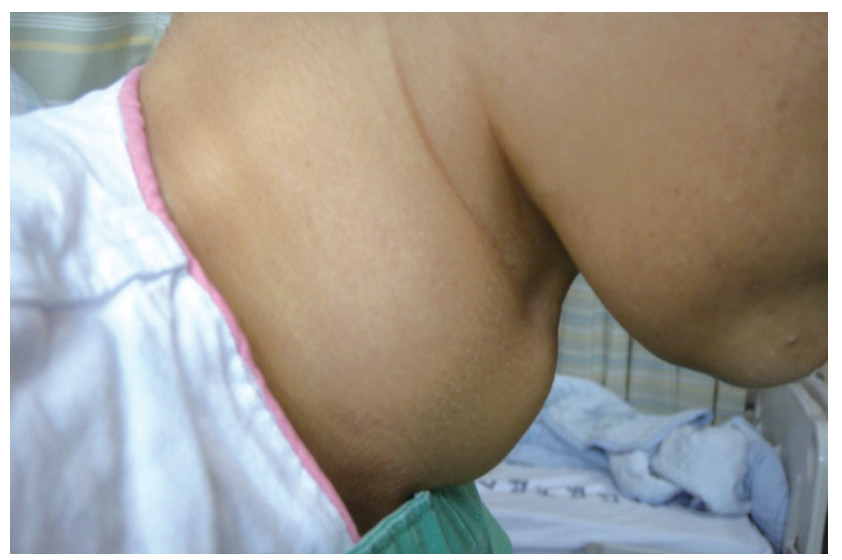

Figure 1. A diffusely swollen and non-tender goiter.

alanine aminotransferase, $66 \mathrm{IU} / \mathrm{L}$; total bilirubin, 4.9 mg/dL; gamma-glutamyl transpeptidase, 984 IU/L; alkaline phosphatase, $694 \mathrm{IU} / \mathrm{L}$; lactate dehydrogenase, 1,301 IU/L; blood urea, $5.5 \mathrm{mg} / \mathrm{dL}$; serum creatinine, $0.4 \mathrm{mg} / \mathrm{dL}$; prothrombin time, 12 seconds; activated partial thromboplastin time, 30.2 seconds; erythrocyte sedimentation rate, $60 \mathrm{~mm}$ /hour; and C-reactive protein, $19.45 \mathrm{mg} / \mathrm{L}$.

Multiple metastatic lesions, including in lymph nodes and the lymphatics of bilateral lung parenchymas and the spine, were confirmed by computed tomography (CT) scanning of the chest. A CT scan of the liver revealed the presence of multiple metastases in the liver parenchyma and ascites. Ascites fluid was aspirated and presented as a transudate. Cytological analysis of the ascites fluid did not reveal the presence of malignant cells.

A magnetic resonance imaging of the brain was done because of sustained headaches. Multiple enhancing masses of variable size with surrounding edema were observed. These findings were consistent with brain metastasis and corresponded to the clinical signs. The patient received whole brain radiation therapy 10 times (300 cGy each treatment). After radiation therapy, the patient received trastuzumab injections ( $8 \mathrm{mg} / \mathrm{kg}$ body weight).

Three days after the trastuzumab injections, the patient complained of a swelling in the anterior neck area (Fig. 1). Laboratory tests to evaluate thyroid function and the presence of thyroid autoantibodies were performed. The test results showed that she was euthyroid with levels of free thyroxine $\left(\mathrm{FT}_{4}, 0.66 \mathrm{ng} / \mathrm{dL}\right.$ [normal range, 0.58 to 1.64]) and thyroid-stimulating hormone (TSH, $3.34 \mu \mathrm{IU} /$ $\mathrm{mL}$ [normal range, 0.34 to 5.6]) within the normal rang-

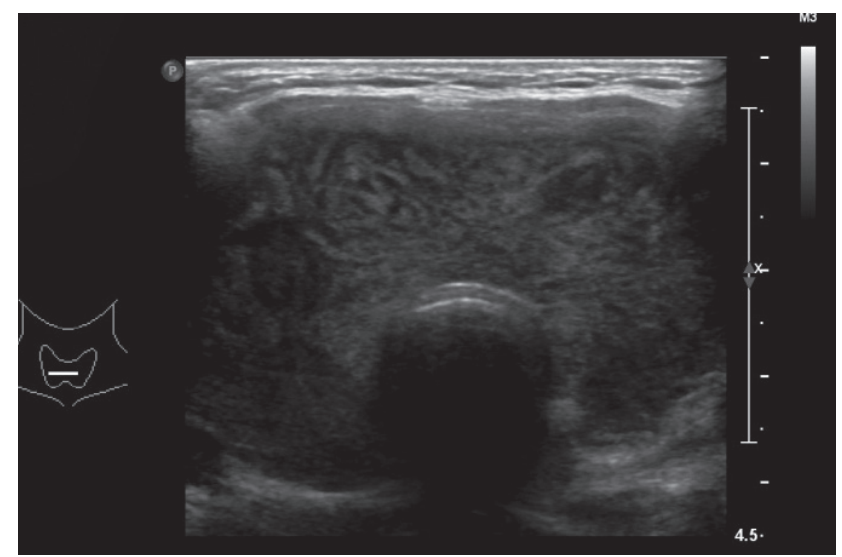

Figure 2. Sonographic imaging showing a diffuse bilateral thyroid gland enlargement without focal lesions and coarse internal echogenicities.

es. Thyroid autoantibodies were also within the normal range, except for the anti-thyroglobulin (TG) antibody (162.6 IU/mL [normal range, o to 40]).

Sonographic imaging revealed that the anterior neck swelling was a diffuse goiter. Internal echogenicity of the bilateral thyroid glands was coarse without focal lesions (Fig. 2). Additionally, a technetium-99m thyroid scan was performed. It showed multiple non-functioning nodules, leading to suspicion of thyroiditis (Fig. 3). We observed her without treatment, and after 5 days, her anti-TG antibody level decreased to $50.63 \mathrm{IU} / \mathrm{mL}$. The follow-up thyroid function tests showed euthyroid status with normal levels of FT4 $(0.76 \mathrm{ng} / \mathrm{dL})$ and TSH (1.69 $\mu \mathrm{IU} / \mathrm{mL})$.

Although radiation therapy and chemotherapy were performed, the general condition of the patient deteriorated. Dyspnea became more prominent as ascites increased, and jaundice developed rapidly. Despite conservative care, the metastatic cancer continued to progress. The patient expired 2 weeks after trastuzumab infusion. The cause of death was intractable respiratory failure and hepatic failure induced by rapidly progressive metastatic breast cancer. From the date of the first trastuzumab infusion to the date of her expiration, other AITD symptoms were not present, except for a palpable goiter.

Currently, chemotherapy, including trastuzumab, is a standard treatment for patients with HER-2-positive breast cancer. Trastuzumab selectively targets breast cancer cells while not affecting normal breast epithelial cells. Therefore, trastuzumab is well tolerated with fewer and milder side effects than conventional chemotherapeutic agents. 


\section{KJIM ${ }^{2}$}

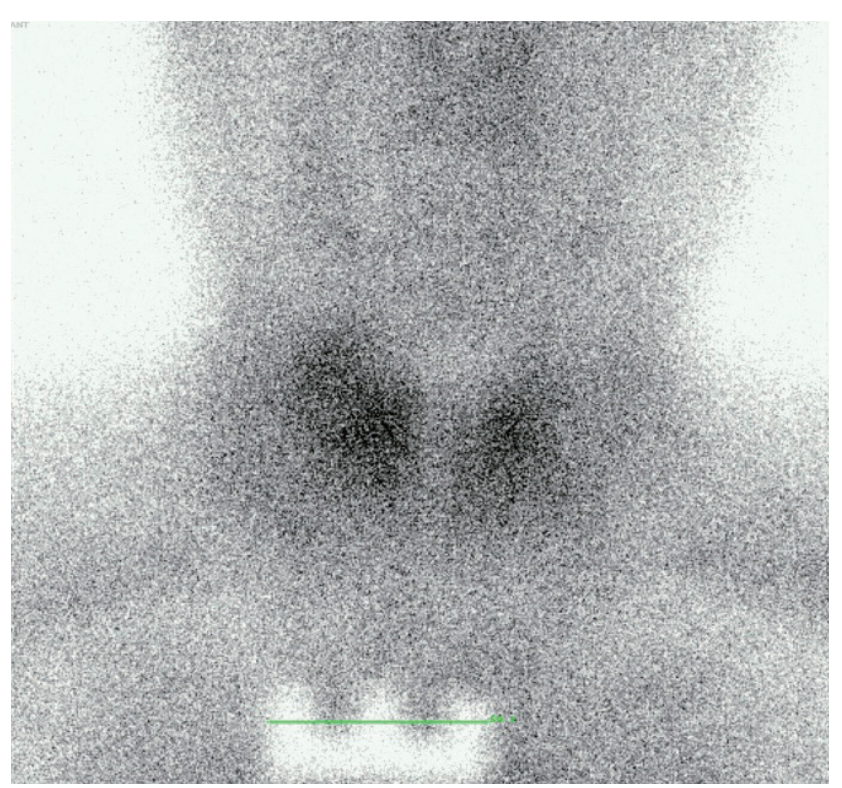

Figure 3. Technetium-99m thyroid scan showing a nonhomogeneous uptake pattern and multiple nonfunctioning thyroid nodules located throughout the bilateral thyroid glands.

According to the U.S. Food and Drug Administration, despite the high tolerability of trastuzumab, several adverse events have been associated with trastuzumab infusion. Autoimmune thyroiditis has been found to be a rare adverse event associated with trastuzumab infusion. Four cases of AITD were reported among 1,678 breast cancer patients who received adjuvant trastuzumab treatment. The median trastuzumab treatment duration in these four cases was 51 weeks, and the incidence rate was $0.3 \%$. Specific details regarding the clinical manifestations, disease severity, disease course, and recurrence were not provided. The likelihood of metastasis to the thyroid gland was low, as the duration between the occurrence of goiter from diagnosis of breast cancer was long, and sonographic findings were not compatible with metastasis.

Targeted therapies including MABs are rapidly being included in the management of many types and stages of cancer. Although the usage of targeted therapies has increased, targeted therapy-associated AITD has been rarely reported. Gefitinib is an inhibitor of HER-1 signalling that is used in the treatment of various solid cancers, including lung and breast cancers. A patient with metastatic squamous cell carcinoma developed vitiligo, an autoimmune mediated skin disorder, after receiving gefitinib for 4 weeks [1]. The underlying mechanisms of targeted therapy-associated AITD are unknown. The immune reconstitution syndrome has been proposed to explain the occurrence of AITD in response to targeted therapy. This hypothesis suggests that agents that influence cellular or humoral immunity may reconstitute the immune system, thereby inducing AITD [2]. Immune modulatory effects of the HER-1 inhibitor involving the control of major histocompatibility complex expression have recently been reported [3], and these immune modulatory effects may play an important role in the occurrence of the HER family inhibitor-associated autoimmune reactions. However, the exact mechanism involved in the development of the HER family inhibitor-associated autoimmune reactions is not fully understood.

AITDs are divided into Graves disease and chronic lymphocytic thyroiditis. Although the clinical manifestations of Graves disease are opposite to those of chronic lymphocytic thyroiditis, these diseases share similar etiologies. Immunological studies have revealed that combined cellular and humoral immune responses affect individuals who are genetically predisposed to develop AITD [4]. A recent study reported that thyropathic women with breast cancer have an increased chance of developing AITD-associated autoantibodies than thyropathic women without breast cancer [5]. The elevated value of anti-TG antibody in our case suggested a pre-existing autoimmune condition. Therefore, we suspected that trastuzumab infusion triggered goiter development in this AITD-susceptible patient.

In our case, the duration between initial administration of trastuzumab and the development of AITD was very short. Furthermore, other possible causative factors or agents for AITD were not identified. Recent studies reported that the HER family of inhibitors may induce autoimmune disorders, and a positive finding of anti-TG antibody indicated that the patient may have been predisposed to autoimmunity. This case is noteworthy because clinical manifestations, severity, immunologic findings, and the natural course of trastuzumab-associated AITD have not been reported previously. In addition, the time frame of the reaction in the current case was extremely short compared to previous reports of HER family inhibitor-associated autoimmune reactions.

Trastuzumab can be used in the treatment of early stage and metastatic breast cancers that overexpress 
HER-2. The recognition of AITD associated with trastuzumab use is difficult, because trastuzumab is usually well tolerated and does not cause marked adverse events. Because thyroid antibodies are more frequently found in thyropathic patients with breast cancer [5], we recommend that clinicians be aware of the possibility of developing AITD in breast cancer patients treated with even a short course of trastuzumab therapy.

Keywords: Breast neoplasms; Trastuzumab; Autoimmune thyroid disease

\section{Conflict of interest}

No potential conflict of interest relevant to this article was reported.

\section{REFERENCES}

1. Jalalat SZ, Cohen PR. Gefitinib-associated vitiligo: report in a man with parotid squamous cell carcinoma and review of drug-induced hypopigmentation. Dermatol Online J 2013;19:20020.

2. Weetman A. Immune reconstitution syndrome and the thyroid. Best Pract Res Clin Endocrinol Metab 2009;23:693702.

3. Pollack BP, Sapkota B, Cartee TV. Epidermal growth factor receptor inhibition augments the expression of MHC class I and II genes. Clin Cancer Res 2011;17:4400-4413.

4. Brown RS. Autoimmune thyroid disease: unlocking a complex puzzle. Curr Opin Pediatr 2009;21:523-528.

5. Grani G, Dicorato P, Dainelli M, et al. Thyroid diseases in women with breast cancer. Clin Ter 2012;163:e401-e404. 